\title{
Quantitative Compositional Stage Mapping and Cluster Analysis Applied to a Vanadian Slag Sample
}

\author{
P. K. Carpenter ${ }^{1}$ \\ 1. Dept. of Earth and Planetary Sciences, Washington University in St. Louis, Saint Louis, MO, USA.
}

Research on rock samples uses both wavelength-dispersive (WDS) and energy-dispersive spectrometry (EDS) for fixed-beam stage mapping on the electron microprobe (EPMA). Recent efforts center on fully quantitative compositional mapping using mean-atomic number (MAN) background correction, with attention to analytical precision, detection limit, accuracy, and downstream processing of map data [1]. The $\mathrm{nA}-\mathrm{sec}$ metric is used for mapping runs where increased probe current compensates for reduced dwell time per pixel. Counting statistics are important for quantitative stage mapping and affect the success of cluster operations in discriminating phase compositions.

A vanadian slag sample is used to illustrate aspects of compositional mapping. The mineralogy is remarkably similar in comparison to $\mathrm{Ca}-\mathrm{Al}$ inclusions in primitive meteorites, and the crystallization sequence represents Fe enrichment during fractional crystallization. Limestone and phosphate added to the molten metal are used to drive steel impurities into the slag during processing, making it silica undersaturated. The slag contains FeNi metal, hibbonite, gehlenite, akermanite, anorthite, silicophosphate, kirschsteinite, and leucite. Fig. 1A shows a color indexed BSE image used to display phases as a locator map, and fig. 1B illustrates the texture and mineralogy in a BSE image. Three distinct gehlenite compositions are present: phenocryst gehlenite which exhibits oscillatory zoning due to Mg-Al substitution, Fe-rich gehlenite forming rims on these phenocrysts as well as late crystallization in the matrix, and vanadium-bearing gehlenite exhibiting significant vanadium substitution. Vanadium is present in coulsonite, augite, and gehlenite, primarily substituting for $\mathrm{Al}^{3+}$ in these phases as $\mathrm{V}^{3+}$ as deduced by stoichiometry, but is also present as both $\mathrm{V}^{3+}$ and $\mathrm{V}^{2+}$ in gehlenite and appears to reflect a change in oxygen fugacity during slag crystallization. The degree of vanadium substitution exceeds that observed in natural minerals and serves to provide insight into the crystal chemistry. Anorthite exhibits spectacular cathodoluminescence zoning (fig 2A) which is likely due to Mn but could also be related to V substitution at trace levels. The zoning features of the gehlenite and anorthite thus record the complex growth and resorption in the slag fraction during processing of the steel.

EPMA quantitative maps were acquired using Probe for EPMA and Probe Image, with two and three pass WDS configurations depending on the elements measured. A BSE image was acquired on each pass to confirm alignment of pixels. CalcImage was used for matrix correction at each pixel and Visual Basic scripts were used to generate color contour maps using Golden Software Surfer (fig. 1C). A Matlab script was used to generate 32-bit tif images and the Fiji Xlib plugin was used for unsupervised k-means cluster calculations and the resulting cluster map is shown in fig $2 \mathrm{~B}$ [2]. The cluster compositions were converted to weight percent phase using the phase area fraction and mineral phase densities. The known compositions of the cluster means were then used to calculate a bulk composition for the sample (fig. 2C). This bulk composition clearly demonstrates the high proportion of $\mathrm{Ca}$ and $\mathrm{Al}$ but also shows that a minor $\mathrm{V}$ concentration can produce significant vanadium substitution.

The textural relations of the slag indicate the crystallization sequence of hibbonite followed by gehlenite and anorthite, then augite. The oxide phase coulsonite is early and Fe gehlenite, leucite, and kirschsteinite 
are late phases, though the slag clearly went through a complex sequence of heating and cooling. Examples of fractional crystallization in geology require reference to suites of rock samples, but this slag sample contains a number of features in one sample that illustrate aspects of crystallization.

\section{References:}

[1] P Carpenter, T Hahn, Microsc. Microanal. 23 (Suppl 1) 2017.

[2] Fiji Xlib plug-in, http://imagej.net/Xlib
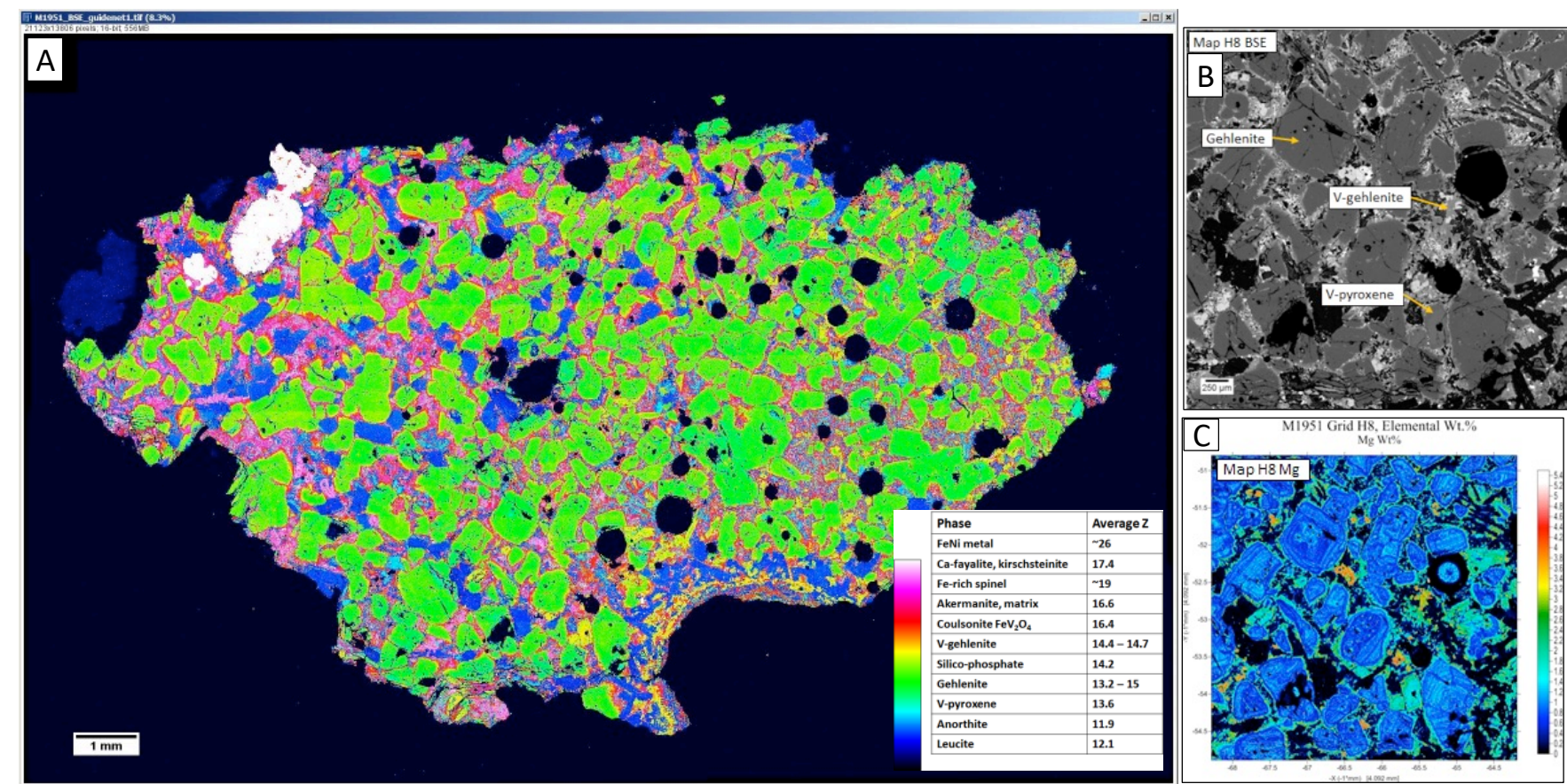

Figure 1. (A) BSE stage map of vanadian slag sample with average Z color lookup table for phase identification. (B) BSE image of detail map from lower center of sample. (C) Quantitative EPMA map of Mg from map H8 showing oscillatory zoning and core-rim zoning.

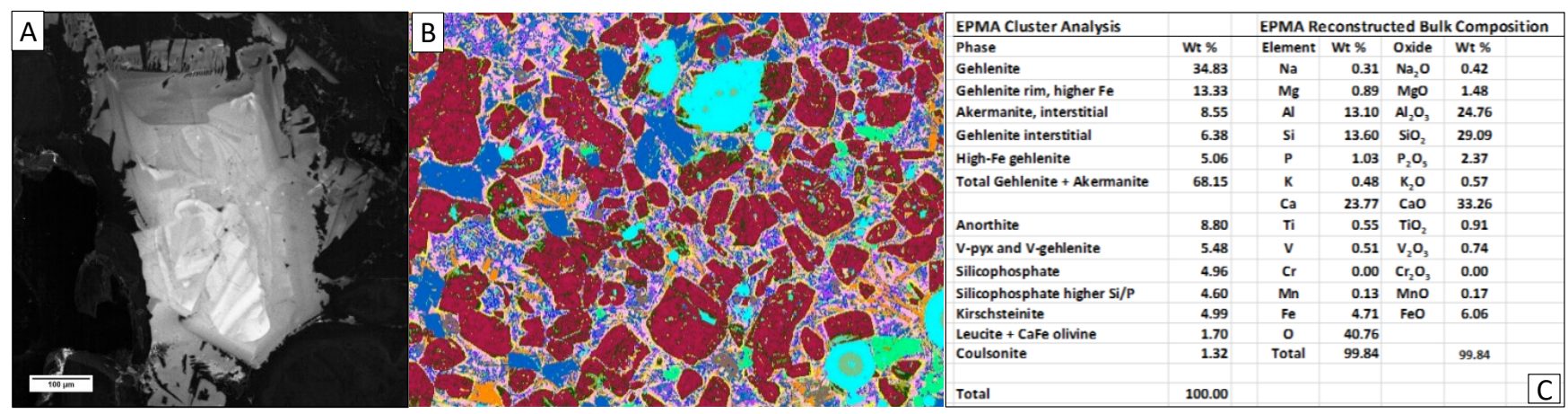

Figure 2. (A) Cathodoluminescence image of anorthite showing complex zoning. (B) Cluster map generated from Fiji Xlib unsupervised k-means clustering. (C) Phase concentrations from cluster data and recalculated EPMA bulk composition for slag sample. 